Research Article

\title{
Parameter Estimation of the Lotka-Volterra Model with Fractional Order Based on the Modulation Function and Its Application
}

\author{
Ying $\mathrm{Hao}^{1,2}$ and Mingshun Guo $\mathbb{D}^{1}$ \\ ${ }^{1}$ School of Management, Shenyang University of Technology, Shenyang 110870, China \\ ${ }^{2}$ Dean's Office, Liaoning Engineering Vocational College, Tieling 112008, China \\ Correspondence should be addressed to Mingshun Guo; syauguo@126.com
}

Received 25 November 2020; Revised 25 May 2021; Accepted 30 June 2021; Published 14 July 2021

Academic Editor: Yakov Strelniker

Copyright (c) 2021 Ying Hao and Mingshun Guo. This is an open access article distributed under the Creative Commons Attribution License, which permits unrestricted use, distribution, and reproduction in any medium, provided the original work is properly cited.

\begin{abstract}
The Lotka-Volterra model is widely applied in various fields, and parameter estimation is important in its application. In this study, the Lotka-Volterra model with universal applicability is established by introducing the fractional order. Modulation function is multiplied by both sides of the Lotka-Volterra model, and the model is converted into linear equations with parameters to be estimated by the fractional integration method. The parameters are obtained by solving the equations. The state of the system is estimated by shifted Chebyshev polynomial. Last, the implementation program of the model is compiled. The concrete implementation method of the improved model is proposed by an example in this study.
\end{abstract}

\section{Introduction}

In the 1940s, Lotka (1925) and Volterra (1926) jointly laid the theoretical foundation for the Lotka-Volterra model. As an extension of the logistic model, this model was used to study the competition and cooperation between different populations in the process of biological evolution. The Lotka-Volterra model is widely applied in the financial industry, resource development, Internet of Things, currency transactions, national economy, food, and other fields [1-8].

To make the Lotka-Volterra model better adapted to applications, a stochastic Lotka-Volterra model disturbed by G-Brownian motion in the framework of nonlinear expectation was proposed by $\mathrm{He}$ et al. [9], which considered the uncertainty of the noise variance. Vadillo [10] studied this problem using three different stochastic models built from a single Lotka-Volterra deterministic model. More concretely, they study their mean-extinction time which satisfies the backward Kolmogorov differential equation, a linear second-order partial differential equation with variable coefficients. An extended Lotka-Volterra model was proposed by Stefan et al. [11], and they studied the microbial dynamics of two interactions in the chemostat. Tahara et al. [12] demonstrated that the Lotka-Volterra system can be stabilized by inducing small immigration of the prey or predator population, and they also checked the effect of the inclusion of the nonlinear interaction term to the stability of the Lotka-Volterra system. Stomov et al. [13] extended the existing $\mathrm{N}$-species impulsive competitive models to an uncertainty case, and the main results are obtained by using Lyapunov-type functions and a comparison principle. Gavina et al. [14] built a Lotka-Volterra competition equation with a nonlinear crowding effect.

Estimating the unknown parameters of the Lotka-Volterra model is a critical issue. A Lotka-Volterra competitive model parameter estimation method based on the grey direct modeling method was proposed by Whu and Wanghai-Jun [15]. Using the least squares method, parameter estimation of a stochastic Lotka-Volterra model driven by small Levy noises from discrete observations was proposed by Wei [16]. A polynomial approximation method 
for the solutions of continuous-time delayed population models was presented by Yuzbasi and Karasayir [17]. They transformed nonlinear algebraic equations for the solution and proposed a technique of residual correction. A regularization method was applied to parameter inversion of the Lotka-Volterra cooperative system with the periodic boundary conditions by $\mathrm{Xu}$ et al. [18]. The unknown parameters of the Lotka-Volterra system can be estimated using the approximate Bayesian computation method by Skvortsov et al. [19]. Perturbed Lyapunov function methods were used to solve multispecies Lotka-Volterra models with regime switching modulated by a continuous-time Markov chain involving a small parameter in Wang et al.'s study [20]. The Lindstedt-Poincare method was applied to the Lotka-Volterra model by Amore and Fernandez [21]. They utilized an efficient systematic algorithm, there were an unprecedented number of perturbation corrections for the two dynamical variables, and the frequency was obtained.

Empirical formulas for complex dynamical processes in some fields are often expressed in the form of power-law functions, and the standard "gradient" law is not satisfied by the corresponding mechanical constitutive relation. Mechanical and physical processes with historical memory and spatial global correlation can be described by fractional differential operators concisely and accurately. In this study, a universal applicability model is established by implanting a fractional-order model into the Lotka-Volterra model. The antinoise property of the algorithm is improved by introducing integrals into the algebraic formula of the variable of the modulation function. The algebraic expression of the algorithm makes it nonasymptotically convergent. Unknown parameter estimation in the Lotka-Volterra model is accurately realized.

\section{Preliminary Knowledge}

2.1. Fractional Calculus. The following notations and definitions are performed before the parameter and system state estimation proof.

Mark: $R^{n}$ represents the $n$-dimensional Euclidean space, and $R^{m \times n}$ represents the $m \times n$ set of real matrices. Given the column vector $x=\left(x_{1}, x_{2}, \ldots, x_{n}\right)^{T} \in R^{n}$, the upper corner " $T$ " represents the transpose. Given that $A \in R^{n \times n}$, $1_{n}=(1,1, \ldots, 1)^{T} \in R^{n}$. Besides, $\operatorname{diag}\left\{d_{1}, d_{2}, \ldots, d_{n}\right\} \in R^{n \times n}$ represents an $n$-order diagonal matrix, and $\operatorname{det}(A)$ represents the determinant of matrix $A$. Given $B \in R^{m \times n}$ and $C=R^{p \times q}, B \otimes C \in R^{m p \times n q}$ represents the Kronecker product of matrix $B$ and matrix $C$. Interval $I=[0, h] \in R, \alpha \in R_{+}$. Moreover, $l=\lfloor\alpha\rfloor+1,\lfloor\alpha\rfloor$ and $\lceil\alpha\rceil$ represent the largest integer which is less than or equal to $\alpha$ and the smallest integer which is more than or equal to $\alpha$, respectively. The $d$ square integrable space of interval $(0, \xi)$ is $\ell^{2}(0, \xi)$.
Definition 1. The left-sided Riemann-Liouville fractional integral of a function $g(\cdot)$ is defined by

$$
\left\{\begin{array}{l}
D_{t}^{0} f(t):=f(t), \\
D_{t}^{\alpha} f(t):=\frac{1}{\Gamma(\alpha)} \int_{0}^{t} \frac{f(\gamma)}{(t-\gamma)^{\alpha-1}} \mathrm{~d} \gamma,
\end{array}\right.
$$

where $\Gamma(\cdot)$ is the gamma function.

It should be noted that $D_{t}^{\alpha} f(t)=D_{t}^{-\alpha} f$ is also used to refer to the $-\alpha$-order Riemann-Liouville fractional derivative of $f$.

Definition 2. The $\alpha$-order left-sided Riemann-Liouville fractional derivative of a function $f(\cdot)$ is defined by

$$
D_{t}^{\alpha} f(t):=\frac{\mathrm{d}^{l}}{\mathrm{~d} t^{l}}\left\{D_{t}^{\alpha-l} f(t)\right\}
$$

Definition 3. The $\alpha$-order left-sided Caputo fractional derivative of a function is defined by

$$
{ }^{C} D_{t}^{\alpha} f(t):=D_{t}^{\alpha-l}\left\{f^{(l)}(t)\right\} .
$$

Definition 4. The right-sided Riemann-Liouville fractional integral of a function $f(\cdot)$ is defined by

$$
\left\{\begin{array}{l}
D_{t, h}^{0} f(t):=f(t) \\
D_{t, h}^{\alpha} f(t):=\frac{(-1)^{l}}{\Gamma(1-\alpha)} \int_{0}^{h} \frac{f^{l}(\gamma)}{(\gamma-t)^{\alpha+1-l}} \mathrm{~d} \gamma
\end{array}\right.
$$

Definition 5. The $\alpha$-order right-sided Riemann-Liouville fractional derivative of a function $f(\cdot)$ is defined by

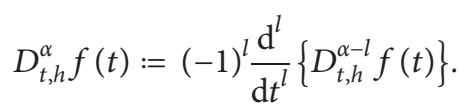

Definition 6 . The $\alpha$-order right-sided Caputo fractional derivative of a function $g(\cdot)$ is defined by

$$
{ }^{C} D_{t}^{\alpha} f(t):=D_{t}^{\alpha-l}\left\{f^{(l)}(t)\right\} \text {. }
$$

Lemma 1. For any interval $[0, h] \in R$, the following formula holds [22]:

$$
\int_{0}^{h} g(t) D_{t}^{\alpha} f(t) \mathrm{d} t=\int_{0}^{h}{ }^{C} D_{t, h}^{\alpha} g(t) f(t) \mathrm{d} t+\sum_{k=0}^{\lceil\alpha\rceil-1}(-1)^{k}\left[g^{(k)}(t) D_{t}^{\alpha-1-k} f(t)\right]_{t=0}^{t=h}
$$




\subsection{Modulation Function}

Definition 7. Given $[a, b] \in R, L \in N^{*}$, if $k=0,1, \ldots, L-1$, then function $g(\cdot)$ meets the criteria $\left.C_{1}\right) g \in C^{L}([a, b])$, $\left.\left.C_{2}\right) g^{(k)}(a)=0, C_{3}\right) g^{(k)}(b)=0$, and the function $g(\cdot)$ is called an $L$-order modulation function on the interval $[a, b]$.

2.3. Shifted Chebyshev Polynomial. Shifted Chebyshev polynomial is defined by the following recursive formula:

$$
\left\{\begin{array}{l}
T_{\xi, 0}(t)=1, \\
T_{\xi, 1}(t)=t, \\
T_{\xi, i+1}(t)=2\left(\frac{2 t}{\xi}-1\right) T_{\xi, i}(t)-T_{\xi, i-1}(t),
\end{array}\right.
$$

Equation (8) is equivalent to the following form:

$$
T_{\xi, i}=i \sum_{k=0}^{i}(-1)^{i-k} \frac{(i+k-1) ! 2^{2 k}}{(i-k) !(2 k) ! \xi^{k}} x^{k} .
$$

Here, $T_{\xi, i}=(-1)^{i}$ and $T_{\xi, i}(\xi)=1$.

Any $x(t) \in \xi^{2}(0, \xi)$ could be represented as a shifted Chebyshev polynomial form as follows:

$$
x(t)=\sum_{j=0}^{\infty} c_{j} T_{\xi, j}(t)
$$

where $\quad c_{j}=1 / h_{j} \int_{0}^{\xi} u(t) T_{\xi, j}(t) \omega_{\xi}(t) \mathrm{d} t, \quad j=1,2, \ldots$ Especially, the preceding $(N+1)$ - paragraph is truncated, and the following can be obtained:

$$
\tilde{x}(t)=\sum_{k=0}^{N} c_{k} T_{\xi, k}(t)
$$

\section{Problem Description and Main Conclusions}

3.1. Problem Description. Assume that multiple species in the same natural environment must affect the survival and development of each other. The fractional-order model is introduced to establish the general applicability Lotka-Volterra model in this study, which is as follows:

$$
\begin{aligned}
D_{t}^{\alpha} x_{i}(t) & =x_{i}(t)\left[b_{i}+\sum_{j=1}^{n} a_{i j} x_{j}(t)\right], \\
x_{i}(t) & =\theta_{i}(t), t \in[0, h]
\end{aligned}
$$

where $t \in I \subset R_{+} U\{0\}, \alpha$ is the fractional order, $0\langle\alpha\rangle 1$, $x_{i(j)}(t)$ is the system state, $\theta(\cdot)$ is the known function, $\theta$ refers to the initialization function of the state $x$, and $a_{i j}$ and $b_{i}$ are the system parameters to be estimated.

Furthermore, the numerical estimates of $x_{i}(t)$ in $t \in(h,+\infty)$ need to be given, $i=1,2, \ldots, n$.
3.2. Parameter and System State Estimation. As a robust and fast estimation method, the modulating functions method has been widely used in the fields of signal processing and automatic control for parameter identification [23], derivative estimation [24, 25], and integral estimation [26]. Recently, it has been extended to state estimation problems for fractional-order systems [27-30]. However, the models considered in previous works are linear. In this study, the modulating functions method is applied for both parameter and state estimation in the nonlinear fractional-order Lotka-Volterra model. First, according to the known initial value function, the unknown parameters $a_{i j}$ and $b_{i}$ of system (12) are estimated, where $i, j=1,2, \ldots, n$. On this basis, the approximate estimation in $t \in[h,+\infty]$ is given.

3.2.1. Parameter Estimation. Before the conclusion is given, $\bar{a}_{i}=\left(a_{i 1}, a_{i 2}, \ldots, a_{i n}, b_{i}\right)^{T}, i=1,2, \ldots, n$. Since system (12) is known at $t \in[0, h]$, estimation of parameters $a_{i j}$ and $b_{i}$ can be transformed into a linear system of equations about these parameters by the modulation function method. Then, $\bar{a}_{i}(i=1,2, \ldots, n)$ can be obtained by selecting the appropriate modulation function.

Proposition 1. Given the known state of system (12), $x_{i}(t)$, $t \in[0, h]$, and if there is a modulation function $g_{i, m}(t)$, then

$$
\operatorname{det}\left(\int_{0}^{h} G_{i}(t) x_{i}(t) X(t) \mathrm{d} t\right) \neq 0 \text {. }
$$

The system parameters $a_{i j}$ and $b_{i}$ can be given by the following formula:

$$
\bar{a}_{i}=\left(\int_{0}^{h} G_{i}(t) x_{i}(t) X(t) \mathrm{d} t\right)^{-1} \int_{0}^{h}{ }^{C} D_{t, t}^{\alpha} g_{i}^{T} x_{i}(t) \mathrm{d} t,
$$

where $\quad x(t)=\left(x_{1}(t), x_{2}(t), \ldots, x_{n}(t)\right)^{T}, \quad \bar{x}(t)=\left(x_{1}(t)\right.$, $\left.x_{2}(t), \cdots, x_{n}(t), 1\right)^{T}, \quad X(t)=1_{n+1} \otimes \bar{x}^{T}(t), \quad g_{i}(t)=\left(g_{i, 1}(t)\right.$, $\left.g_{i, 2}(t), \ldots, g_{i, n}(t), g_{i, n+1}(t)\right)^{T}, \quad G_{i}(t)=\operatorname{diag}\left\{g_{i, 1}(t), g_{i, 2}\right.$ $\left.(t), \ldots, g_{i, n}(t), g_{i, n+1}(t)\right\}, \quad i, j=1,2, \ldots, n, \quad$ and $n, m=$ $1,2, \cdots, n+1$.

Proof. Multiply $g_{i, m}(t)$ in both sides of equation (12) and the integrals from 0 to $h$. Obtain $i=1,2, \ldots$ and $n, m=1,2, \ldots, n+1$.

$$
\int_{0}^{h} g_{i, m}(t) D_{t}^{\alpha} x_{i}(t) \mathrm{d} t=\int_{0}^{h} g_{i, m}(t) x_{i}(t)\left[b_{i}+\sum_{j=1}^{n} a_{i j} x_{j}(t)\right] \mathrm{d} t .
$$

According to Lemma 1, the left side of equation (16) becomes

$$
\int_{0}^{h} g_{i, m}(t) D_{t, h}^{\alpha} g_{i, m}(t) x(t) \mathrm{d} t+\sum_{k=0}^{\lceil\alpha\rceil-1}(-1)^{k}\left[g_{i, m}^{(k)}(t) D_{t}^{\alpha-1-k} x_{i}(t)\right]_{t=0}^{t=h} .
$$

According to Definition 7, from formula (17), the following equation can be obtained: 


$$
\int_{0}^{h} g_{i, m}(t) D_{t}^{\alpha} x_{i}(t) \mathrm{d} t=\int_{0}^{h}{ }^{C} D_{t, h}^{\alpha} g_{i, m}(t) x_{i}(t) \mathrm{d} t
$$

where $I=1,2, \ldots$ and $n, m=1,2, \ldots, n+1$. Therefore, according to formula (18),

$$
\left(\int_{0}^{h} G_{i}(t) x_{i}(t) X(t) \mathrm{d} t\right) \bar{a} i=\int_{0}^{h} \mathrm{CD}_{t, h}^{\alpha} g_{i, m}^{T}(t) x_{i}(t) \mathrm{d} t .
$$

Thus, Proposition 1 can be proved.

\subsubsection{State Estimation. Suppose}

$$
C=\left[\begin{array}{cccc}
c_{10} & c_{20} & \cdots & c_{n 0} \\
c_{11} & c_{21} & \cdots & c_{n 1} \\
\vdots & \vdots & \ddots & \vdots \\
c_{1 N} & c_{2 N} & \cdots & c_{n N}
\end{array}\right] .
$$

According to the Chebyshev polynomial, $\tilde{x}_{i}(t)=\sum_{k=0}^{N} c_{i} k T_{\xi, k}(t)$ can be used to estimate the corresponding system state $x_{i}(t), i=1,2, \ldots$ in $t \in(h,+\infty)$ approximately, that is, estimates of the system state $x_{i}(t)$, $t \in(h,+\infty)$, can be obtained by solving the algebraic equation $C$ ( $c_{i k}$ can be obtained based on the known system state in $t \in[0, h])$.

Proposition 2. If there are $N+1$ time points, then $t_{0}, t_{1}, \cdots, t_{N} \in[0, h]$ becomes

$$
\begin{gathered}
\operatorname{det}\left(T_{\xi}\right) \neq 0 \\
\text { where } T_{\xi}=\left[\begin{array}{cccc}
T_{\xi, 0}\left(t_{0}\right) & T_{\xi, 1}\left(t_{0}\right) & \cdots & T_{\xi, N}\left(t_{0}\right) \\
T_{\xi, 0}\left(t_{1}\right) & T_{\xi, 1}\left(t_{1}\right) & \cdots & T_{\xi, N}\left(t_{1}\right) \\
\vdots & \vdots & \ddots & \vdots \\
T_{\xi, 0}\left(t_{N}\right) & T_{\xi, 1}\left(t_{N}\right) & \cdots & T_{\xi, N}\left(t_{N}\right)
\end{array}\right] .
\end{gathered}
$$

Then, the state of system (12) $x_{i}(t), t \in(h,+\infty)$ can be approximately given by $\tilde{x}_{i}(t)=\sum_{k=0}^{N} c_{i} k T_{\xi, k}(t)$, and $c_{i k}(i=$ $1,2, \ldots, n, k=1,2, \ldots, N)$ can be given by the following formula:

$$
\begin{gathered}
C=T_{\xi}^{-1} \theta \\
\text { where } \theta=\left[\begin{array}{cccc}
\theta_{1}\left(t_{0}\right) & \theta_{2}\left(t_{0}\right) & \cdots & \theta_{n}\left(t_{0}\right) \\
\theta_{1}\left(t_{1}\right) & \theta_{2}\left(t_{1}\right) & \cdots & \theta_{n}\left(t_{1}\right) \\
\vdots & \vdots & \ddots & \vdots \\
\theta_{1}\left(t_{N}\right) & \theta_{2}\left(t_{N}\right) & \cdots & \theta_{n}\left(t_{N}\right)
\end{array}\right] \text {. }
\end{gathered}
$$

Proof. According to formula (11), the numerical solution of the system state $x_{i}(t), t \in(h,+\infty)$ can be obtained only by solving the corresponding coefficient $c_{i k}(i=1,2, \ldots$, $n, k=1,2, \ldots, N)$.

According to the known conditions and formula (13), the following can be obtained:

$$
x_{i}\left(t_{q}\right)=\theta_{i}\left(t_{q}\right), \quad q=0,1, \ldots, N .
$$

The first $(N+1)$ term $\tilde{x}_{i}(t)$ is used to approximate $x_{i}(t)$, and then, system (23) is

$$
\sum_{k=0}^{N} c_{i k} T_{\xi, k}(t q)=\theta_{i}\left(t_{q}\right), \quad q=0,1, \ldots, N,
$$

that is,

$$
T_{\xi} C=\theta
$$

Thus, according to formula (21), the proposition is proved.

\section{Example Analysis}

The survival and development of different populations in the same natural environment must influence each other. The degree of interaction among multiple populations can be analyzed quantitatively by the Lotka-Volterra model under the premise of a certain population size $(n)$. The Lotka-Volterra model with fractional-order multicompetition relations in this study has more general application. The corresponding examples are discussed for $n=3$ and $n=2$, respectively.

Example 1. The Lotka-Volterra competition model is a dynamic model, and the cooperative relationship between enterprises can be reflected by this model [31]. Three companies with more invention patents in the new energy vehicle industry are chosen by this study. The number of patent holdings in the future can be predicted by the Lotka-Volterra model with the fractional order. In order to meet the requirements of the algorithm for sample data, the patent dates of enterprises are fitted to make the data continuous. Equation (12) can be written as

$$
D_{t}^{\alpha} x_{i}(t)=x_{i}(t)\left[b_{i}+\sum_{j=1}^{3} a_{i j} x_{j}(t)\right], \quad i=1,2,3,
$$

where $x_{i}(t)$ is the patent number of enterprise $i, x_{j}(t)$ is the patent number of enterprise $j, b_{i}$ is the change rate of each enterprise's own patents, and $a_{i j}$ is the influence intensity of enterprise $j$ on enterprise $i$.

The distribution of the patent holdings of the three enterprises in the past five years is given in Table 1.

According to the parameter identification (as shown in Figure 1), the number of patent granted to the Zhejiang Geely Holding Group Co., Ltd. is influenced mostly by BYD Company Limited, and the number of patent granted to Beijing New Energy Automobile Co., Ltd. is influenced least by BYD Company Limited. The number change of patent granted of the three enterprises is less affected by the enterprises themselves and more affected by other enterprises in the same system. In addition, $a_{12}<0, a_{13}<0, a_{21}>0$, and $a_{31}<0$; it shows that in terms of patent granted number, BYD Company Limited and Zhejiang Geely Holding Group Co., Ltd. are in the predator-prey system and BYD Company Limited and Beijing New Energy Automobile Co. Ltd. are in the mutualism system.

Figure 2 shows that the growth trend of the patent holdings of BYD Company Limited has a significant slowing trend while patent holdings of Zhejiang Geely Holding 
TABLE 1: Distribution of patent holdings in recent years.

\begin{tabular}{|c|c|c|c|c|c|}
\hline Enterprise & 2014 & 2015 & 2016 & 2017 & 2018 \\
\hline BYD Company Limited & 1320 & 2589 & 4199 & 5517 & 6067 \\
\hline Zhejiang Geely Holding Group Co., Ltd. & 652 & 1085 & 1796 & 2338 & 3080 \\
\hline Beijing New Energy Automobile Co., Ltd. & 219 & 701 & 1568 & 2330 & 2801 \\
\hline
\end{tabular}

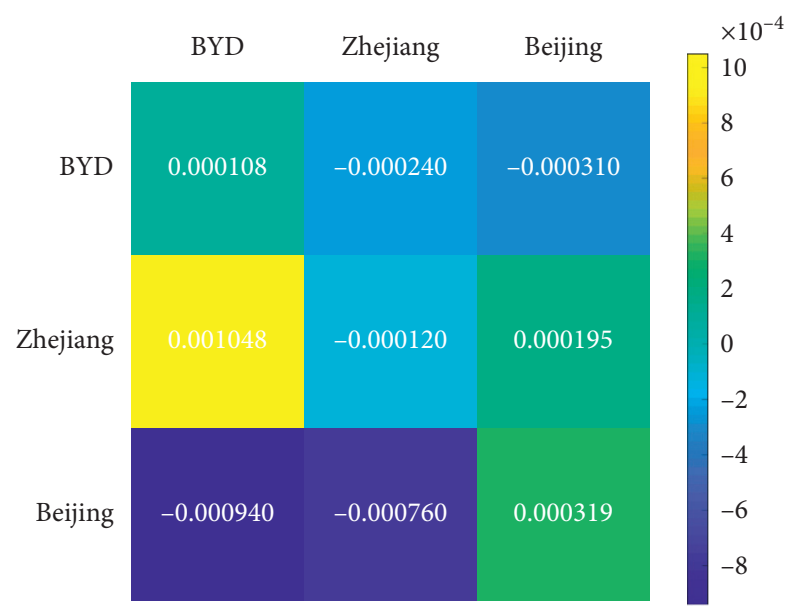

FIGURE 1: Results of parameter identification.

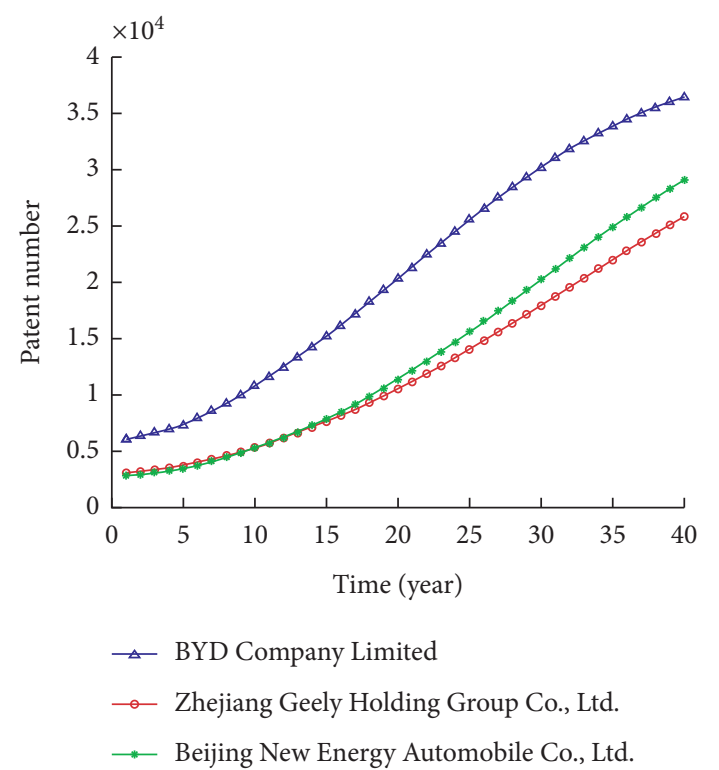

FIGURE 2: Forecasting results of enterprise patent quantity for the next 30 years.

Group Co., Ltd. and Beijing New Energy Automobile Co., Ltd. are in a period of rapid growth after 10 years. Patent holdings of BYD Company Limited are influenced by another two enterprises increasingly seriously. Moreover, the patent holdings of Beijing New Energy Automobile Co. Ltd. will surpass Zhejiang Geely Holding Group Co., Ltd. in five years. The gap between patent holdings of the two enterprises will widen as time goes on.

Example 2. Research on parameter estimation of patent application number of two competitive enterprises is in order to reflect the relationship between patent applied number and patent granted number more objectively. The insufficiency of decision condition in Example 1 is supplemented. Equation (12) can be written as

$$
D_{t}^{\alpha} x_{i}(t)=x_{i}(t)\left[b_{i}+\sum_{j=1}^{2} a_{i j} x_{j}(t)\right], \quad i=1,2 \text {, }
$$

where $x_{i}(t)$ is the patent applied number of enterprise $i$, $x_{j}(t)$ is the patent applied number of enterprise $j, b_{i}$ is the patent application change rate of each enterprise, and $a_{i j}$ is the influence intensity of enterprise $j$ on enterprise $i$.

Patents applied number of the two enterprises from 2014 to 2018 is given in Table 2 .

According to the parameter identification (as shown in Figure 3), in terms of the patent application number, BYD Company Limited and Zhejiang Geely Holding Group Co., Ltd. are in the predator-prey system. The change of the patent applied number of Zhejiang Geely Holding Group Co., Ltd. is influenced mostly by BYD Company Limited, while the influence of it owns much less. It shows that the innovation ability of Zhejiang Geely Holding Group Co., Ltd. is slightly lower than BYD Company Limited, and BYD Company Limited has a leading edge in technology. 
TABLE 2: Distribution of patent applications in recent years.

\begin{tabular}{lccccc}
\hline Enterprise & 2014 & 2015 & 2016 & 2017 & 2018 \\
\hline BYD Company Limited & 1843 & 1923 & 3197 & 3209 & 2597 \\
$\begin{array}{l}\text { Zhejiang Geely Holding Group } \\
\text { Co., Ltd. }\end{array}$ & 1452 & 859 & 1555 & 1276 & 2800 \\
\hline
\end{tabular}

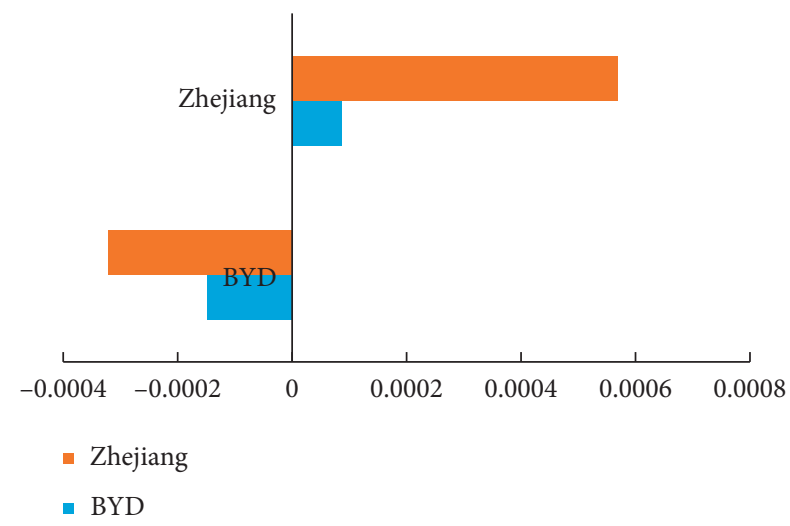

FIGURE 3: Results of parameters estimation.

\section{Conclusion}

In this study, the modulating functions method was applied to estimate unknown parameters, and the state of the Lotka-Volterra model was defined by a fractional-order nonlinear system. First, by multiplying the modulating functions to the considered model and applying fractionalorder integration by parts formulas, the differential equations can be transformed into a set of integral equations where the unknown parameters are linear concerning the integrals. Then, the unknowns are estimated by solving the linear system of the obtained integral equations. Second, by taking the truncated expansion of the state based on the shifted Chebyshev polynomials, the unknown coefficients are obtained by solving a linear system using the initialization function of the state. Then, these estimated coefficients are used to predict the future behavior of the state.

\section{Data Availability}

The data used to support the findings of this study are available from the corresponding author upon request.

\section{Conflicts of Interest}

The authors declare that they have no conflicts of interest.

\section{Acknowledgments}

This work was supported by the Humanities and Social Sciences Fund of Department of Education of Liaoning Province, China (LGZY2019004), the Special Project of Serving Local Economic and Social Development DecisionMaking of Shenyang University of Technology (FWDFGD2020010), and the Humanities and Social Sciences Fund of Liaoning Engineering Vocational College, China (ZYS201901).

\section{References}

[1] S. J. Lee, D. J. Lee, and H. S. Oh, "Technological forecasting at the Korean stock market: a dynamic competition analysis using Lotka-Volterra model," Technological Forecasting and Social Change, vol. 72, pp. 1004-1057, 2015.

[2] L. Lambertini and G. Leitmann, "On the attainment of the maximum sustainable yield in the Verhulst-Lotka-Volterra model," Automatica, vol. 110, Article ID 108555, 2019.

[3] R. Casado-Vara, A. Canal-Alonso, M. D. Rey et al., "Smart buildings ioT networks accuracy evolution prediction to improve their reliability using a Lotka-Volterra Ecosystem Model," Sensors, vol. 19, no. 21, Article ID 4642, 2019.

[4] P. Gatabazi, J. C. Mba, and E. Pindza, "Modeling cryptocurrencies transaction counts using variable-order fractional grey Lotka-Volterra dynamical system," Chaos, Solitons and Fractals, vol. 127, pp. 283-290, 2019.

[5] I. Ivanova, O. Strand, and L. Leydesdorff, "An eco-systems approach to constructing economic complexity measures: endogenization of the technological dimension using LotkaVolterra equations," Advances in Complex Systems, vol. 22, no. 1, Article ID 1850023, 2019.

[6] L. Shi, Y. Xu, L. Zhan et al., "Enhancing vitamin B12 content in co-fermented soy-milk via a Lotka Volterra model," Turkish Journal of Biochemistry, vol. 43, no. 6, pp. 671-678, 2018.

[7] Z. Cao, Q. Zhang, and Y. Zhao, "The principle of competitive exclusion about a stochastic lotka-volterra model with two predators competing for one prey," Discrete Dynamics in Nature and Society, vol. 2018, Article ID 7312581, 13 pages, 2018.

[8] H.-C. Hung, Y.-C. Chiu, H.-C. Huang et al., "An enhanced application of Lotka-Volterra model to forecast the sales of two competing retail formats," Computers and Industrial Engineering, vol. 109, pp. 325-334, 2017.

[9] P. He, Y. Ren, and D. Zhang, "Asymptotic moment estimation for stochastic Lotka-Volterra model driven by G -Brownian motion," Stochastics An International Journal of Probability and Stochastic Processes, vol. 2020, Article ID 3705325, 18 pages, 2020.

[10] F. Vadillo, "Comparing stochastic Lotka-Volterra predatorprey models," Applied Mathematics and Computation, vol. 360, pp. 181-189, 2019.

[11] V. Stefan, D. B. Sophie, F. Karoline et al., "Bistability in a system of two species interacting through mutualism as well as competition: chemostat vs. Lotka-Volterra equations," PLoS One, vol. 13, no. 6, Article ID e0197462, 2018.

[12] T. Tahara, M. K. A. Gavina, T. Kawano et al., "Asymptotic stability of a modified Lotka-Volterra model with small immigrations," Entific Reports, vol. 8, Article ID 7029, 2018.

[13] G. T. Stamov, S. Simeonov, and I. M. Stamova, "Uncertain impulsive Lotka-Volterra competitive systems: robust stability of almost periodic solutions," Chaos, Solitons and Fractals: Applications in Science and Engineering: An Interdisciplinary Journal of Nonlinear Science, vol. 110, pp. 178184, 2018.

[14] M. K. A. Gavina, T. Tahara, K. I. Tainaka et al., "Multi-species coexistence in Lotka-Volterra competitive systems with crowding effects," Scientific Reports, vol. 8, Article ID 1198, 2018.

[15] L. F. Wu and Y. N. WangHai-jun, "Estimation the parameters of Lotka-Volterra model based on the grey direct modeling and its application," Expert Systems with Applications, vol. 38, no. 6, pp. 6412-6416, 2011. 
[16] C. Wei, "Parameter estimation for stochastic Lotka-Volterra model driven by small Levy noises from discrete observations," Communication in Statistics-Theory and Methods, vol. 12, pp. 1-10, 2020.

[17] S. Yuzbasi and M. Karacayir, "A numerical method for solutions of Lotka-Volterra predator-prey model with timedelay," International Journal of Biomathematics, vol. 11, no. 2, Article ID 1850028, 2018.

[18] L. Xu, J. Y. Liu, and G. Zhang, "Pattern formation and parameter inversion for a discrete Lotka-Volterra cooperative system," Chaos Solitons and Fractals, vol. 110, pp. 226-231, 2018.

[19] A. Skvortsov, B. Ristic, and A. Kamenev, "Predicting population extinction from early observations of the LotkaVolterra system," Applied Mathematics and Computation, vol. 320, pp. 371-379, 2018.

[20] R. Wang, X. Y. Li, and G. Yin, "Asymptotic properties of multi-species lotka-volterra models with regime switching involving weak and strong interactions," Journal of Nonlinear Science, vol. 30, no. 2, pp. 565-601, 2020.

[21] P. Amore and F. M. Fernandez, "On the application of the Lindstedt-Poincare method to the Lotka-Volterra system," Annals of Physics, vol. 396, pp. 293-303, 2018.

[22] Y. Q. Podlubny and Y. Chen, "Adjoint fractional differential expressions and operators," in Proceedings of ASME International Design Engineering Technical Conferences and Computers and Information in Engineering Conference IDETC/CIE, pp. 4-7, Las Vegas, NV, USA, September 2007.

[23] D. Y. Liu, T. M. Laleg-Kirati, O. Gibaru, and W. Perruquetti, "Identification of fractional order systems using modulating functions method," in Proceedings of the 2013 American Control Conference, pp. 17-19, Washington, DC, USA, June 2013.

[24] D. Y. Liu, T. M. Laleg-Kirati, W. Perruquetti, and O. Gibaru, "Non-asymptotic state estimation for a class of linear timevarying systems with unknown inputs," in Proceedings of the 19th World Congress of the International Federation of Automatic Control, pp. 324-328, Cape Town, South Africa, August 2014.

[25] D. Y. Liu and T. M. Laleg-Kirati, "Robust fractional order differentiators using generalized modulating functions method," Signal Processing, vol. 107, pp. 395-406, 2015.

[26] Y. Tian, Y. Q. Wei, D. Y. Liu, and D. Boutat, "Fast and robust estimation for positions and velocities from noisy accelerations using generalized modulating functions method," $\mathrm{Me}$ chanical Systems and Signal Processing, vol. 133, Article ID 106270, 2019.

[27] X. Wei, D. Y. Liu, and D. Boutat, "Non-asymptotic state estimation for a class of fractional order linear systems," IEEE Transactions on Automatic Control, vol. 62, no. 3, pp. 11501164, 2016.

[28] Y. Q. Wei, D. Y. Liu, D. Boutat, and Y. M. Chen, "An improved pseudo-state estimator for a class of commensurate fractional order linear systems based on fractional order modulating functions," Systems and Control Letters, vol. 118, pp. 29-34, 2018.

[29] Y. Q. Wei, D. Y. Liu, and D. Boutat, "Innovative fractional derivative estimation of the pseudo-state for a class of fractional order linear systems," Automatica, vol. 99, pp. 157-166, 2019.

[30] Y. Q. Wei, D. Y. Liu, D. Boutat, H. R Liu, and C. Lv, "Modulating functions based differentiator of the pseudostate for a class of fractional order linear systems," Journal of
Computational and Applied Mathematics, vol. 384, Article ID 113161, 2021.

[31] D. Guo, H. Zhang, C. Zheng et al., "Analysis of the future development of Chinese auto energy saving and environmental benefits," Systems Engineering-- Theory\&Practice, vol. 36, no. 6, pp. 1593-1599, 2016. 